\title{
Exact Results for the Asymmetric Simple Exclusion Process with a Blockage*
}

\author{
S. A. Janowsky ${ }^{\dagger \ddagger}$ and J. L. Lebowitz \\ Departments of Physics and Mathematics \\ Rutgers University \\ New Brunswick, New Jersey 08903
}

June 1993

\begin{abstract}
We present new results for the current as a function of transmission rate in the one dimensional totally asymmetric simple exclusion process (TASEP) with a blockage that lowers the jump rate at one site from one to $r<1$. Exact finite volume results serve to bound the allowed values for the current in the infinite system. This proves the existence of a gap in allowed density corresponding to a nonequilibrium "phase transition" in the infinite system. A series expansion in $r$, derived from the finite systems, is proven to be asymptotic for all sufficiently large systems. Padé approximants based on this series, which make specific assumptions about the nature of the singularity at $r=1$, match numerical data for the "infinite" system to a part in $10^{4}$.
\end{abstract}

\section{Introduction}

The one dimensional totally asymmetric simple exclusion process (TASEP) is a continuous-time stochastic process in which particles on a one dimensional lattice jump independently and randomly at unit rate to vacant neighboring sites on their immediate right [1]. It corresponds to a Kawasaki exchange dynamics [2] at infinite temperature and infinite electric field [3]. The stationary state of this system for $N$ particles on a ring of $K$ sites, $K \geq N$, gives equal weight to all $\left(\begin{array}{l}K \\ N\end{array}\right)$ permissible configurations. This measure goes over, in the limit $K \rightarrow \infty, N / K \rightarrow \rho$, to the product measure

\footnotetext{
${ }^{*}$ Supported in part by NSF Grant DMR92-13424

${ }^{\dagger}$ Supported in part by NSF Mathematical Sciences Postdoctoral Research Fellowship DMS 90-07206

${ }^{\ddagger}$ Address after August 1993: Department of Mathematics, University of Texas, Austin, TX 78712
} 
with occupation probability $\rho$. The TASEP is thus the simplest driven diffusive lattice-gas model whose dynamics does not satisfy detailed balance [3]. It is also, for the infinite lattice, an example of a microscopic system from which one can derive Euler-like hydrodynamical equations 泪, e.g. the Burgers equation.

In an earlier work [5] we introduced a variant of the TASEP where the jump rate across one bond of the system was reduced from 1 to $r, 0<r<1$. If one thinks of the TASEP as a model for fluid flow in a pipe, this is analogous to a restriction in the diameter of the pipe. More realistically perhaps we could consider a superionic conductor [6] like $\mathrm{AgI}$ or $\mathrm{KAg}_{4} \mathrm{I}_{5}$, in the geometry of a pinched doughnut, with a time-varying magnetic field generating an electromotive force; or a road under construction in a model of traffic flow [7]. Clearly the rate decrease will increase the particle density to the immediate left of this "blockage" bond and decrease the density to its immediate right, but what is not obvious is that this perturbation may have global in addition to local effects. Equivalent to introducing a defect into a growth surface [8, 9], this blockage can cause the nonequilibrium stationary states of the model to exhibit a segregation into high- and low-density regions. This allows the full complexity of the model, previously available only through time-dependent studies, to be displayed via the stationary state [5, 10]. Among other features we observed for the system of $N$ particles on a ring of size $K$, with $N$ and $K$ large, was the equivalence of the exponent governing the time and space scaling of shock fluctuations.

It is convenient to label the sites on the ring from $-K / 2$ to $K / 2(-K / 2$ and $K / 2$ refer to the same site), with the blockage located at the bond between 0 and 1 . The stationary density profile for a half-filled system can be seen in fig. 1. As we approach the limit $K \rightarrow \infty$ keeping $N / K=\rho$ fixed, we have that far from the blockage the density becomes uniform and the stationary state appears to be asymptotically a product measure [11] with densities $\lim _{x \rightarrow \pm \infty} \rho(x)=\rho_{ \pm}$, with $\rho_{+}<\rho_{-}$, independent of $\rho$, for $|\rho-1 / 2|$ less than a certain value described in the next section. The densities are related by the condition of constant current: $J=\rho_{+}\left(1-\rho_{+}\right)=\rho_{-}\left(1-\rho_{-}\right)$. When segregation takes place we thus have $1 / 2-\rho_{+}=\rho_{-}-1 / 2$. One is of course interested in the asymptotic densities $\rho_{ \pm}$and current $J$ as a function of the transmission rate $r$. In [5] these could only be determined numerically; the 


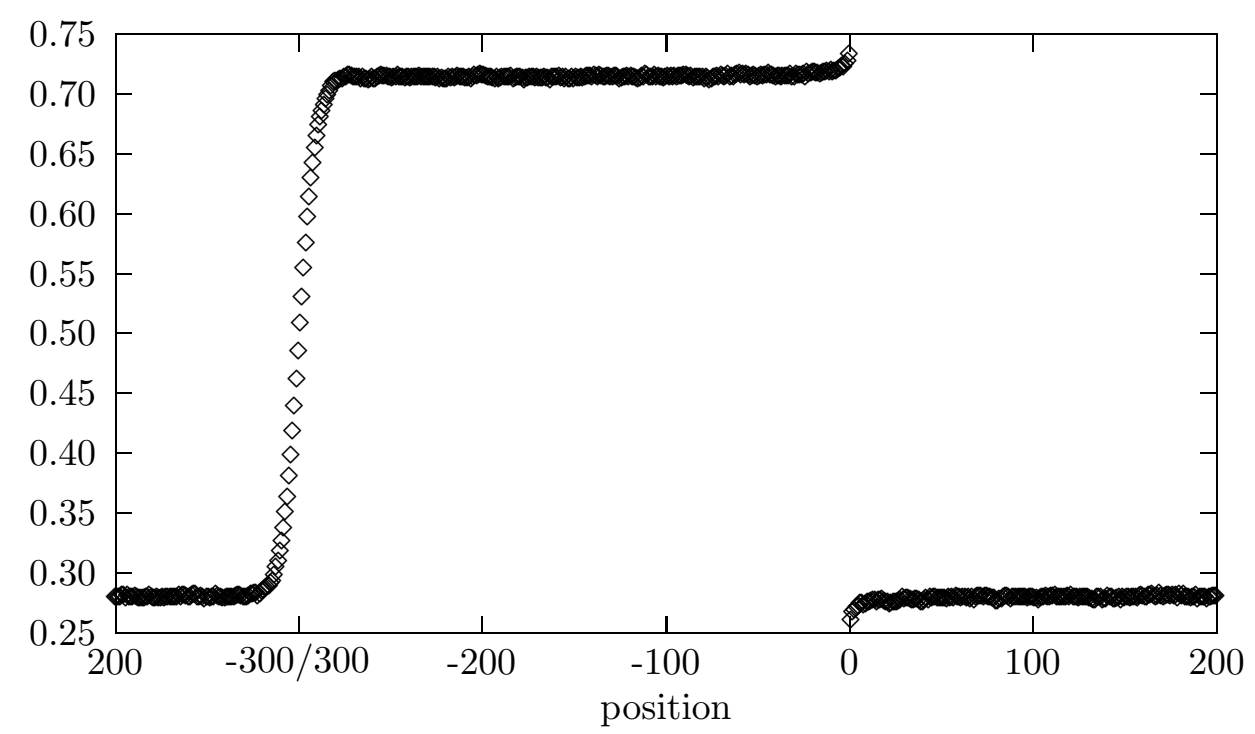

Figure 1: Density profile (from time average in simulation) for a half-filled system with 600 sites and periodic boundary conditions. The blockage bond is located between site 0 and site 1 and has the value $r=0.33$.

simple estimate obtained by neglecting the (large) correlations near the blockage, i.e. by assuming a uniform product measure with density $\rho_{-}\left(\right.$resp. $\left.\rho_{+}\right)$to the left (resp. right) of the origin, gives

$$
J \approx r \rho_{-}\left(1-\rho_{+}\right) \approx \frac{r}{(1+r)^{2}},
$$

which is accurate only for very small $r$. This is in contrast with the translation invariant case, where, as already mentioned, there are no correlations in the stationary state. Here, in order to obtain more accurate estimates, it is necessary to include the effects of correlations, which decay slowly with the distance from the blockage, typical of systems with conservative dynamics not satisfying detailed balance [12, 13, 14]. (In [5] this decay was found numerically to go like the inverse power of the distance.)

Although one is interested in asymptotic behavior, it can be fruitful to examine the behavior of small systems with open boundary conditions for which one can determine the stationary state exactly. In a closely related model [15], this led to an exact solution for all system sizes. Furthermore, 
as we shall see, results for such systems give a systematic improvement on the estimate (11) as well as bounds on the current $J$ for the infinite system at fixed $r$ and thus also on the maximum value of $\rho_{+}$. We therefore studied systems, ranging in size from 2 to 10 sites, in which particles are added at the left with rate $\alpha$ and removed at the right with rate $\beta$. At non-boundary sites particles jump independently to empty neighboring sites on the right with rate $r$ for the jump between sites 0 and 1 , where the blockage is located, and with rate 1 for all other jumps. The process is thus defined (on a lattice with $K=2 L$ sites) by the generator $\mathcal{L}$ giving the rate of change of any function of the configuration $\eta=\{\eta(-L+1), \eta(-L+2), \ldots, \eta(L)\}$, where $\eta(k)=0$ or 1 is the occupation number at site $k$ :

$$
\begin{aligned}
\mathcal{L} f(\eta)= & \alpha\left[f\left(\eta_{-L+1,+}\right)-f(\eta)\right]+\sum_{i=-L+1}^{-1}\left[f\left(\eta_{i \rightarrow i+1}\right)-f(\eta)\right] \\
& +r\left[f\left(\eta_{0 \rightarrow 1}\right)-f(\eta)\right]+\sum_{i=1}^{L-1}\left[f\left(\eta_{i \rightarrow i+1}\right)-f(\eta)\right] \\
& +\beta\left[f\left(\eta_{L,-}\right)-f(\eta)\right],
\end{aligned}
$$

where $\eta_{i \rightarrow j}(k)$ gives the configuration at site $k$ after an attempted jump from site $i$ to site $j$ :

$$
\eta_{i \rightarrow j}(k)= \begin{cases}1, & k=j \text { and } \eta(i)=1 \\ 0, & k=i \text { and } \eta(j)=0 \\ \eta(k), & \text { otherwise }\end{cases}
$$

the boundary terms are given in terms of

$$
\eta_{i, \pm}(k)= \begin{cases}(1 \pm 1) / 2, & k=i \\ \eta(k), & k \neq i\end{cases}
$$

\section{Exact Solutions For Small Systems}

For a system with $2 L$ sites there are $2^{2 L}$ possible configurations. By considering the set of equations $\left\langle\mathcal{L} f_{i}\right\rangle=0$ where $f_{i}$ is the characteristic function of the $i$ th configuration, one obtains a system of equations whose (normalized) solution is the unique stationary state for the model, i.e. one obtains the unique stationary solution of the master equation for the open finite system. 
Solving such a system of equations is a daunting computational task. However, by fixing $\alpha$ and $\beta$ and then making use of the reflection symmetry of the problem (which for $\alpha=\beta$ reduces the number of variables by a factor slightly less than 2) we were able to exactly solve for systems up to size 8 leaving $r$ as an indeterminate parameter and size 10 for fixed $r$. The computations were performed using Maple V running on a SPARCstation 2.

Typically we took $\alpha=\beta=1$; the asymptotic behavior should be independent of $\alpha$ and $\beta$ provided they are greater than some critical value which goes to zero as $r$ goes to zero and equals $1 / 2$ for $r \geq 1$. This critical value is simply related to the maximum current the infinite system with blockage $r$ can support; it is just the density of a product measure at the maximum current: $\left(1-\sqrt{1-4 J_{\max }}\right) / 2$. Clearly $J_{\max }(r)$ is a monotone nondecreasing function of $r$ equal to $1 / 4$ for $r \geq 1$. The system "selects" the state of maximum current, $\rho_{+}=\left(1-\sqrt{1-4 J_{\max }}\right) / 2$ and $\rho_{-}=1-\rho_{+}$, if the boundaries can supply and remove particles quickly enough [16], so the (asymptotic) state should not depend on the precise values of $\alpha$ and $\beta$; corrections to this asymptotic behavior will be local to the boundary region [15, 17]. Alternatively, if one considered periodic boundary conditions instead of an open system we would expect equivalent asymptotics with densities $\rho_{-}=1-\rho_{+}$for all values of the average density $\rho$ between $\left(1-\sqrt{1-4 J_{\max }}\right) / 2$ and $\left(1+\sqrt{1-4 J_{\max }}\right) / 2$. For average densities outside this interval the limiting asymptotic densities should be equal to the average density both to the left

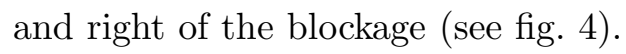

We present some of the results for small systems below. $J_{L}(r, a)$ is the steady state current in a system with $2 L$ sites at $\alpha=\beta=a ; J_{L}(r) \equiv J_{L}(r, 1)$. We have

$$
\begin{gathered}
J_{1}(r, a)=\frac{2 a r}{2 a+3 r}, \quad J_{1}(r)=\frac{2 r}{2+3 r}, \quad J_{1}(r, 1 / 2)=\frac{r}{1+3 r} \\
J_{2}(r, a)=2 a r\left[4 a^{2}+8 a^{3}+4 a^{4}+\left(7 a+12 a^{2}+6 a^{3}\right) r+\left(2+4 a+2 a^{2}\right) r^{2}\right] / \\
{\left[8 a^{3}+16 a^{4}+8 a^{5}+\left(14 a^{2}+36 a^{3}+36 a^{4}+12 a^{5}\right) r\right.} \\
\left.+\left(18 a+45 a^{2}+44 a^{3}+18 a^{4}\right) r^{2}+\left(5+14 a+14 a^{2}+6 a^{3}\right) r^{3}\right] \\
J_{2}(r)=\frac{2 r\left(16+25 r+8 r^{2}\right)}{32+98 r+125 r^{2}+39 r^{3}}, \quad J_{2}(r, 1 / 2)=\frac{2 r\left(9+29 r+18 r^{2}\right)}{18+85 r+215 r^{2}+130 r^{3}}
\end{gathered}
$$




$$
\begin{aligned}
J_{3}(r)=4 & \left(47775744+261095424 r+669424384 r^{2}+1009680576 r^{3}+968982368 r^{4}\right. \\
& \left.+609395274 r^{5}+250834237 r^{6}+65287925 r^{7}+9784215 r^{8}+644781 r^{9}\right) / \\
( & 191102976+1331036160 r+4447316992 r^{2}+9277942272 r^{3} \\
& +12731145304 r^{4}+11671707972 r^{5}+7170513506 r^{6}+2914237861 r^{7} \\
+ & \left.753023405 r^{8}+112354075 r^{9}+7383541 r^{10}\right) .
\end{aligned}
$$

We observe that $J_{L}(r)$ is a rational function of $r$ with integer coefficients, and the order of the function and the complexity of the coefficients grow rapidly with system size $-J_{4}(r)$ has terms up to 34 th order with the largest coefficient being 51 digits long, and is reproduced in Appendix A. We note that the value of $J_{L}(1)$ was obtained explicitly in 15 for all $L$, namely that

$$
J_{L}(1)=\frac{1}{4}+\frac{3}{4(1+4 L)}=J_{\infty}(1)\left[1+\frac{3}{1+4 L}\right]
$$

\section{Bounds On The Infinite System}

For $r=1$, product measure with any density $\rho \in[0,1]$ is stationary in the infinite system, and thus the system can have any current in the range 0 to $1 / 4$. For $r<1$, this is not necessarily the case; $J_{\max }(r)$ may be less than $1 / 4$. In this case only a range of densities satisfying $\left|\rho_{ \pm}-1 / 2\right|>\sqrt{1-4 J_{\max }(r)} / 2$ is permitted. The problem thus is to find bounds on $J_{\max }(r)$. A very simple bound on $J_{\max }(r)$ can be obtained by noting that if we remove the right (or left) half of the system we are left with a system of $L$ sites with input (removal) rate $a$ and removal (input) rate $r$. Calling $\hat{J}(L ; \alpha, \beta)$ the current in a system of $L$ sites with input rate $\alpha, \operatorname{removal} \operatorname{rate} \beta$, and all "internal" jump rates now being unity, we clearly have

$$
J_{L}(r, a) \leq \hat{J}(L ; a, r)=\hat{J}(L ; r, a)
$$

where the last equality, as well as exact formulae for $\hat{J}(L ; \alpha, \beta)$, have been computed in 15, 17]. In particular for $a \geq r$ and $r \leq 1 / 2$ we have $\lim _{L \rightarrow \infty} \hat{J}(L ; \alpha, r)=r(1-r)$ so that $J_{\max }(r) \leq r(1-r)$ for $r \leq 1 / 2$. 


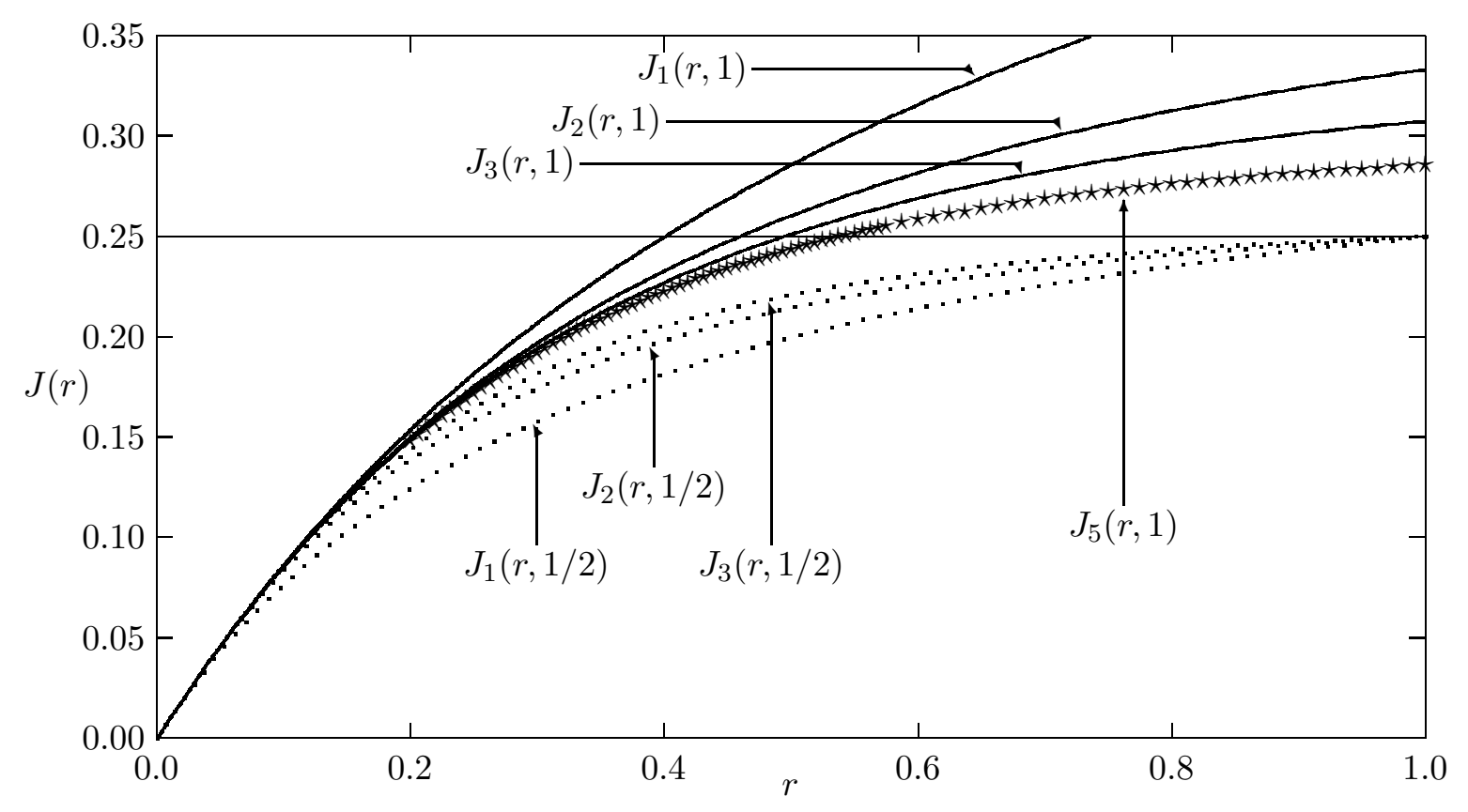

Figure 2: $J_{L}(r, 1)$ and $J_{L}(r, 1 / 2)$ for several values of $L$. Where $J_{L}(r, 1)<1 / 4$ there must be a gap in the allowed stationary measures of the infinite system.

For $a$ and $r$ both greater than $1 / 2$ the right hand side of (10) approaches $1 / 4$ so it yields no new information-just a proof that $J_{\max }(r)$ cannot exceed $1 / 4$.

To obtain better bounds we note that for any configuration, since the maximum rate at which a particle attempts to jump on to any site is bounded by one, the current in a system of size $L$ with boundary conditions $\alpha=\beta=1$ cannot increase as $L$ increases. Note that $\alpha=\beta=1$ corresponds to keeping the site $-L$ always occupied and the site $L+1$ always vacant in a system of size $L^{\prime}>L$. Thus $J_{L}(r)$ is monotonically decreasing in $L$, and for every finite value of $L, J_{L}(r)$ is an upper bound for $J_{\infty}(r)$. (Similar arguments show that $J_{L}(r, a)$ is nondecreasing in $r$ and $a$.) These bounds prove the existence of a gap in the set of stationary measures of the infinite system; the results for finite $L$ (illustrated in fig. 2) show that wherever there exists an $L$ such that $J_{L}(r)<1 / 4$ there is a forbidden range of currents. (One result from our simulations is that $J_{15000}(0.8)=0.24979(5)$, indicating that the gap exists at least up to $r=0.8$.) 
We note here also that letting $a \rightarrow \infty$ in $J_{L}(r, a)$ corresponds to reducing the size of the system from $2 L$ to $2(L-1)$ so that $\lim _{a \rightarrow \infty} J_{L}(r, a)=J_{L-1}(r)$. Using monotonicity in $a$ this implies again that $J_{L}(r)$ is monotone nonincreasing in $L$.

For the case $\alpha=\beta=1 / 2$ the sequence $J_{L}(r, 1 / 2)$ appears to be increasing in $L$, and we believe but cannot prove that $J_{\infty}(r) \geq J_{L}(r, 1 / 2)$. Proving such an inequality is nontrivial since $J_{\infty}(r)$ is the maximum current the infinite system can have; there exist stationary measures where the current is less than $J_{L}(r, 1 / 2)$. Thus our results for finite $L$ only provide rigorous one-sided bounds. Note also that $J_{L}(1,1 / 2)=1 / 4$ for all $L$, c.f. 15].

\section{Series expansion}

While there is no apparent pattern to the "raw" expressions for the current (5)-(8), (A.1), one does emerge if we examine a Taylor expansion around $r=0$. We obtain:

$$
\begin{aligned}
J_{1}(r)= & r-\frac{3}{2} r^{2}+\frac{9}{4} r^{3}-\frac{27}{8} r^{4}+\frac{81}{16} r^{5}-\frac{243}{32} r^{6}+O\left(r^{7}\right), \\
J_{2}(r)= & r-\frac{3}{2} r^{2}+\frac{19}{16} r^{3}-\frac{257}{256} r^{4}+\frac{24105}{4096} r^{5}-\frac{829297}{65536} r^{6}+O\left(r^{7}\right), \\
J_{3}(r)= & r-\frac{3}{2} r^{2}+\frac{19}{16} r^{3}-\frac{21535}{27648} r^{4}+\frac{919407829}{214990848} r^{5}-\frac{7398899579671}{417942208512} r^{6}+O\left(r^{7}\right), \\
J_{4}(r)= & r-\frac{3}{2} r^{2}+\frac{19}{16} r^{3}-\frac{21535}{27648} r^{4}+\frac{77729356627}{146767085568} r^{5} \\
& +\frac{1067903077191004635349}{126214320739011526656} r^{6}+O\left(r^{7}\right) .
\end{aligned}
$$

There is a clear pattern: as we increase the size of the system the low-order coefficients stop changing after a certain point. Assuming the continuation of this behavior, and including the results for the size 10 system, we have

$$
\begin{aligned}
J_{\infty}(r) & =r-\frac{3}{2} r^{2}+\frac{19}{16} r^{3}-\frac{21535}{27648} r^{4}+\frac{77729356627}{146767085568} r^{5}-.3278724755(1) r^{6}+O\left(r^{7}\right) \\
& =r-\frac{3}{2} r^{2}+\frac{19}{2^{4}} r^{3}-\frac{5 \cdot 59 \cdot 73}{2^{10} \cdot 3^{3}} r^{4}+\frac{13 \cdot 33613 \cdot 177883}{2^{26} \cdot 3^{7}} r^{5}-.3278724755(1) r^{6}+O\left(r^{7}\right) ;
\end{aligned}
$$


in the second part of (15) we show the prime factorization of the coefficients; the denominators appear deceptively simple while examination of the numerators proves less instructive.

Including the dependence of the boundary terms (i.e. taking $a \neq 1$ ) does not significantly alter this behavior:

$$
\begin{aligned}
J_{1}(r, a)= & r-\frac{3}{2 a} r^{2}+\frac{9}{4 a^{2}} r^{3}-\frac{27}{8 a^{3}} r^{4}+O\left(r^{5}\right) \\
J_{2}(r, a)= & r-\frac{3}{2} r^{2}+\frac{9 a^{4}+18 a^{3}+7 a^{2}-8 a-7}{4 a^{2}(a+1)^{2}} r^{3} \\
& -\frac{54 a^{7}+216 a^{6}+300 a^{5}+64 a^{4}-294 a^{3}-370 a^{2}-188 a-39}{16 a^{3}(a+1)^{4}} r^{4}+O\left(r^{5}\right) \\
J_{3}(r, a)= & r-\frac{3}{2} r^{2}+\frac{19}{16} r^{3}+\left[514 a^{14}+6939 a^{13}+41551 a^{12}+144387 a^{11}+316671 a^{10}\right. \\
& +432661 a^{9}+285181 a^{8}-176743 a^{7}-702157 a^{6}-944908 a^{5}-799104 a^{4}-457504 a^{3} \\
& \left.-172480 a^{2}-38528 a-3840\right] r^{4} /\left[256 a^{3}(1+2 a)(a+2)^{3}(a+1)^{7}\right]+O\left(r^{5}\right)
\end{aligned}
$$

The dependence on the boundary appears one term earlier than in (11)-(14), but otherwise the structure is the same.

In fact, the assumption regarding the behavior of the Taylor coefficients for progressively larger systems expressed in (11)-(18) can be proven to be correct:

Theorem 1 Fix $L_{2}$. Then for $L_{1} \leq L_{2}, J_{L_{2}}(r)=J_{L_{1}}(r)+O\left(r^{L_{1}+2}\right)$.

Theorem 2 Fix $L_{2}$. Then for $L_{1} \leq L_{2}$ and $a>0, J_{L_{2}}(r, a)=J_{L_{1}}(r)+O\left(r^{L_{1}+1}\right)$.

Thus we see a small system not only bounds but also provides a good approximation (at least for small $r$ ) for any (finite) system that is larger. This also strongly suggests that the approach of $J_{L}(r)$ to $J_{\infty}(r)$ is exponential for $r<1$. Of course the rate of the exponential approach vanishes at $r=1$, where the convergence becomes algebraic (see (99)). We reserve the proof for Appendix B. 


\section{Padé Approximants}

The Taylor series given in (15) gives an accurate measure of the current for small $r$, but for large $r$ it is less successful. In fact, we can see that the series must break down by $r=1$ : although one commonly thinks of $r$ as being a transmission rate $\leq 1$, it is perfectly acceptable to take $r>1$ in the generator (2). It is also fairly easy to see, by comparison with a system with a boundary at the origin, that the infinite volume current $J_{\infty}(r)$ will have the same value for all $r \geq 1$, namely $J_{\infty}(1)=1 / 4$, so that there must be a nonanalyticity for some $r \leq 1$ in $J_{\infty}(r)$. One can also examine the coefficients of (15) and see that they apparently decrease (in magnitude) rather slowly, indicating that the radius of convergence of the series is most probably 1 . Some numerical analysis indicates that there is no discontinuity in any of the derivatives of $J_{\infty}(r)$ at $r=1$; this evidence leads us to hypothesize that there is an essential singularity at $r=1$ in $J_{\infty}(r)$. An alternative which cannot be ruled out by our results is that $J_{\infty}(r)=1 / 4$ for $r>r_{1}$ with $r_{1}<1$. Numerical results for the structure of $J_{L}(r)$ would seem however to argue in favor of a changeover at $r=1$.

We thus look for a function of the appropriate form for the current. The "simplest" function with an essential singularity at $r=1$ that also gives $J_{\infty}(0)=0$ and $J_{\infty}(1)=1 / 4$ is a function of the form

$$
J_{\infty}(r)=1 / 4-\exp [f(r)] / 4
$$

where $f(r)$ has a simple pole at $r=1$. We therefore examined functions of the form

$$
f(r)=\frac{1}{4}-\frac{1}{4} \exp \left[\frac{p(r)}{(1-r) q(r)}\right]
$$

specifically, we used our Taylor series to fit the following Padé approximants:

$$
\begin{aligned}
J^{33}(r) & =\frac{1}{4}-\frac{1}{4} \exp \left[\frac{-4 r\left(1+a_{1} r+a_{2} r^{2}\right)}{(1-r)\left(1+b_{1} r+b_{2} r^{2}\right)}\right], \\
J^{43}(r) & =\frac{1}{4}-\frac{1}{4} \exp \left[\frac{-4 r\left(1+a_{1}^{\prime} r+a_{2}^{\prime} r^{2}+a_{3}^{\prime} r^{3}\right)}{(1-r)\left(1+b_{1}^{\prime} r+b_{2}^{\prime} r^{2}\right)}\right], \\
J^{34}(r) & =\frac{1}{4}-\frac{1}{4} \exp \left[\frac{-4 r\left(1+a_{1}^{\prime \prime} r+a_{2}^{\prime \prime} r^{2}\right)}{(1-r)\left(1+b_{1}^{\prime \prime} r+b_{2}^{\prime \prime} r^{2}+b_{3}^{\prime \prime} r^{3}\right)}\right] .
\end{aligned}
$$




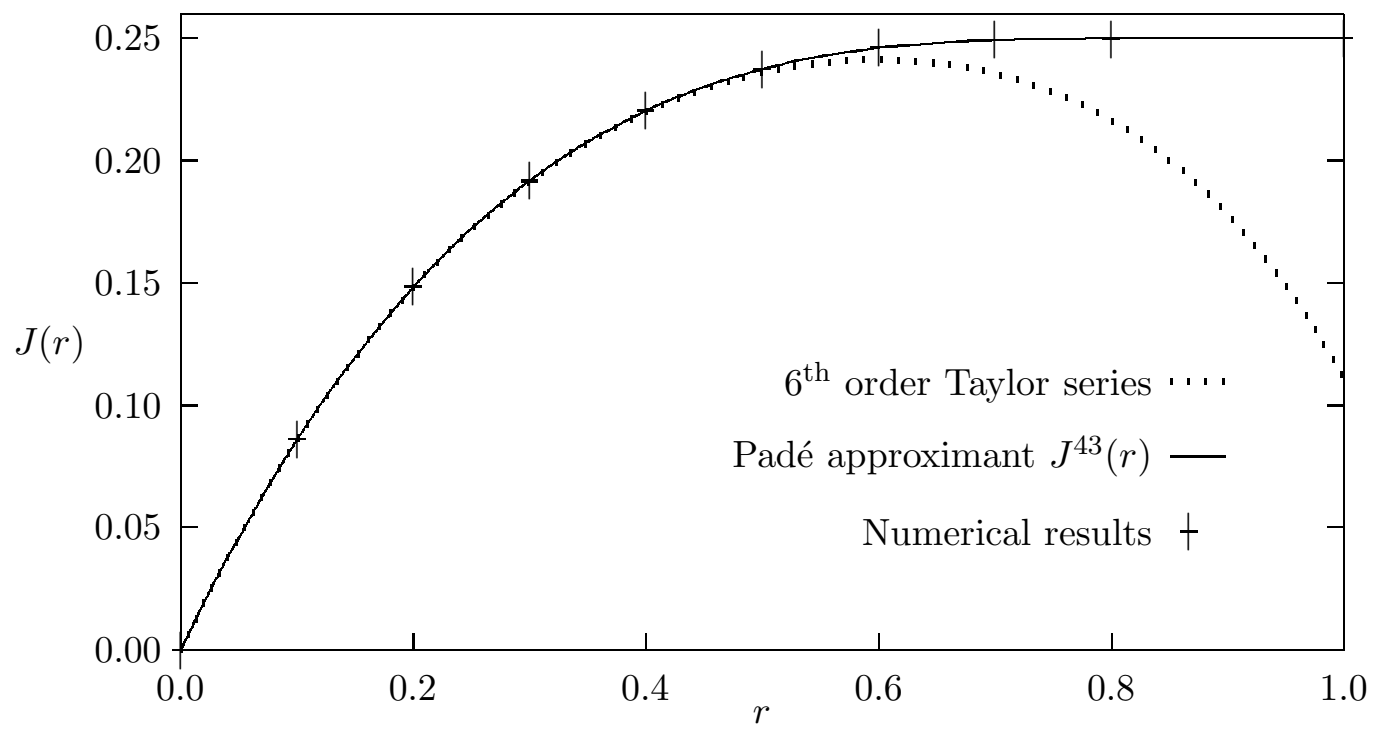

Figure 3: Taylor, Padé and numerical results. Estimated errors for the numerical calculations are less than the thickness of the lines, ranging from $5 \times 10^{-5}$ to $2 \times 10^{-4}$.

The actual values of the coefficients of the Padé approximants are not particularly revealing, as one might expect. The Padé functions do, however, appear to be converging pointwise: for $r \in[0,1]$ the maximum difference between any of $J^{33}, J^{43}$ and $J^{34}$ is less than $2 \times 10^{-5}$.

Of course we are not interested in how well the different approximants approximate each other, but how well they approximate $J_{\infty}(r)$. We thus must compare the approximants with numerical simulations. We see in fig. 3 that the approximation is within the error bounds of the simulations; we happened to have plotted $J^{43}$ but any of the approximants would have fit the data as well. In comparison, a Padé approximant that behaves quadratically or quartically at $r=1$ (as opposed to exponentially) looks qualitatively similar but does not fall within the error bounds of the simulations.

The simulation results were obtained by direct simulation of the TASEP dynamics; progressively larger systems were used until the current reached an asymptotic value. For small values of $r$ this occurred quite quickly, but the needed system size grows quite rapidly as one approaches $r=1$ : for $r=0.7$ we needed to investigate systems with 6400 sites and for $r=0.8$ we needed to investigate 


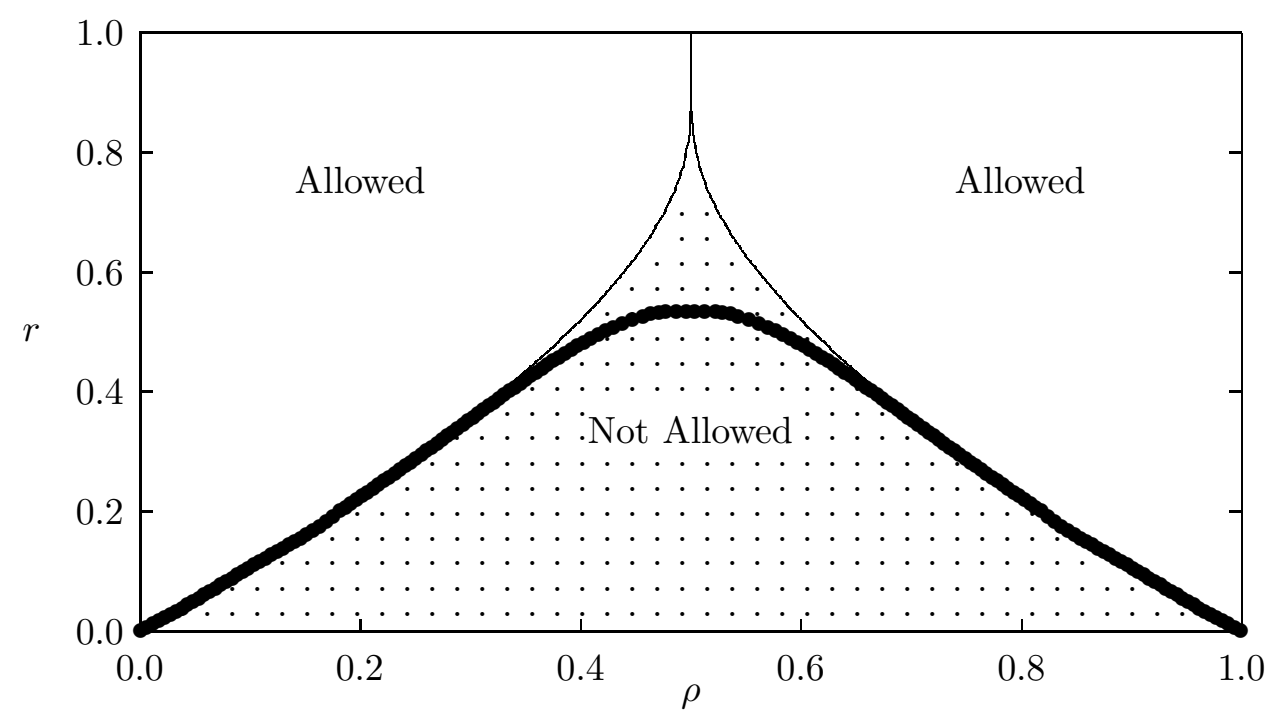

Figure 4: Phase diagram of allowed asymptotic density vs. transmission. The heavy line indicates the region excluded by currently available exact results; the thin line and the shaded region are determined by the Padé results.

systems with 15000 sites.

Our results can also be viewed as the determination of the phase diagram for the system. As mentioned earlier the current $J_{\infty}(r)$ is the maximum stationary current permitted by the infinite system - with some boundary conditions the current may be less. Accepting [1] that the stationary measure is asymptotically a product measure then $J_{\infty}=\rho_{\infty}\left(1-\rho_{\infty}\right)$, and the bound on the current is equivalent to a bound on the allowable range of densities. We plot the boundary of this range, the critical density, against the transmission rate in fig. 4 . If the overall density of the finite system in a periodic box, $\rho$, was in the disallowed region, the system would have to segregate with high density $\rho_{-}$to the left of the blockage and low density $\rho_{+}$to the right of it, with $\rho_{-}+\rho_{+}=1$ and $\rho=c \rho_{-}+(1-c) \rho_{+}$for some fraction $c, 0<c<1$.

The phase diagram is apparently quite different from that normally observed in systems that phase segregate, with a cusp at the critical point if one believes the Padé approximants accurately 
describe the behavior of the system. Of course one should remember the rather unique nature of this type of transition.

\section{Discussion}

When a system has dynamics that do not satisfy detailed balance, the steady states do not (in general) have the form of Gibbs states. Thus, even a qualitative description of the nature of the steady states, particularly with respect to the dependence on the parameters entering the dynamics, is lacking. Standard perturbative techniques are generally inapplicable, and most of our knowledge comes from computer simulations, approximate calculations using renormalization group (universality) ideas, and a few exactly soluble models.

It thus is quite remarkable that information determined from a system with 10 sites can be used to predict the behavior of a system with $10^{4}$ sites to within a part in $10^{4}$ for all values of the parameter $r$. This is particularly interesting given that quantities besides the asymptotic current, e.g. the local density near the blockage, are not given accurately by the results from the small systems.

We hope that it will be possible to determine a general formula for the terms in our Taylor seriescertainly the sequence of denominators $\left(1,2,2^{4}, 2^{10} 3^{3}, 2^{26} 3^{7}\right)$ seems tractable. Since the coefficients are independent of system size (if the system is big enough) only reasonably-sized systems need to be studied in order to obtain useful results. Unfortunately direct computation of higher-order terms seems unlikely, given that the computational complexity grows exponentially, without further theoretical input.

Even if our computations cannot be extended, there remains the possibility of proving that some of our qualitative description of the phase diagram is correct. Our series is only proven to be asymptotic for small $r$, but we have already seen that finite systems can serve to bound certain properties of the infinite system for arbitrary values of $r$ : for example we believe that $J_{L}(r, 1 / 2) \leq J_{\infty}(r)$ and we know that $J_{\infty}(r) \leq J_{L}(r, 1)$ for all $L>0$ and all $0 \leq r \leq 1$. 


\section{Appendix A The function $J_{4}(r)$}

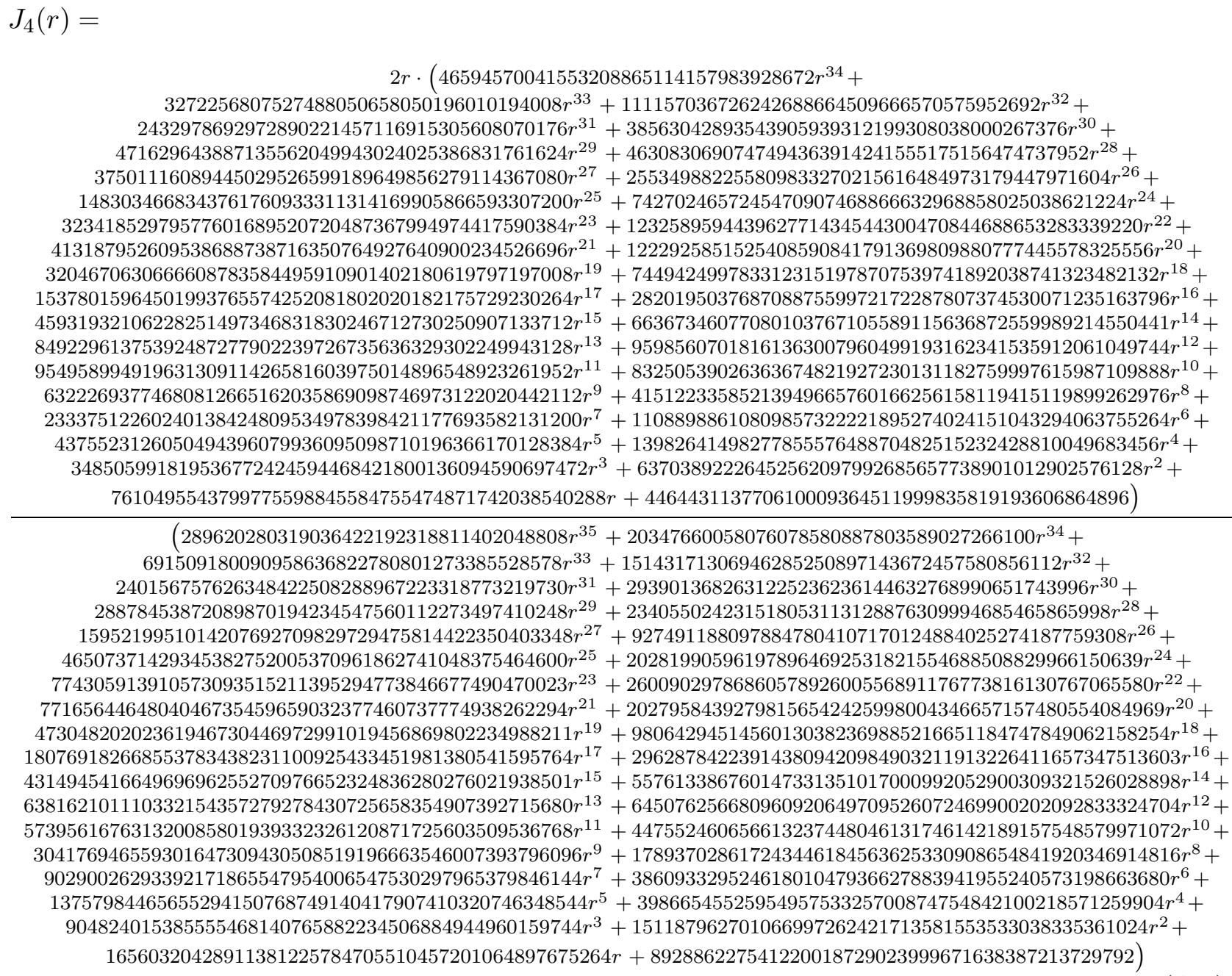

\section{Appendix B Proof of Theorem [1}

To prove theorem 1, we will need to consider systems with unequal numbers of sites to the left and to

the right of the blockage, say $L_{-}$and $L_{+}$, respectively; $J_{L_{-}, L_{+}}(r)$ will represent the current in such a system with $\alpha=\beta=1$. We will prove the following lemma:

Lemma 1 For $L_{-}^{\prime} \leq L_{-}$and $L_{+}^{\prime} \leq L_{+}$,

$$
J_{L_{-}, L_{+}}(r)=J_{L_{-}^{\prime}, L_{+}}(r)+O\left(r^{L_{-}^{\prime}+2}\right) \quad \text { and } \quad J_{L_{-}, L_{+}}(r)=J_{L_{-}, L_{+}^{\prime}}(r)+O\left(r^{L_{+}^{\prime}+2}\right) .
$$


Theorem 1 is a direct consequence: for $L_{2} \geq L_{1}, J_{L_{2}}(r) \equiv J_{L_{2}, L_{2}}(r)=J_{L_{2}, L_{1}}(r)+O\left(r^{L_{1}+2}\right)=$ $J_{L_{1}, L_{1}}(r)+O\left(r^{L_{1}+2}\right) \equiv J_{L_{1}}(r)+O\left(r^{L_{1}+2}\right)$.

Now we prove the lemma, considering only the case $L_{+}^{\prime} \leq L_{+}$since the case $L_{-}^{\prime} \leq L_{-}$is the same by symmetry. We will need to consider probabilities of certain collections of configurations. We write

$$
\operatorname{Pr}\left(\eta_{-L_{-}+1}, \eta_{-L_{-}+2}, \ldots, \eta_{0} ; \eta_{1}, \eta_{2}, \ldots, \eta_{N}\right)_{L_{-}, L_{+}^{\prime}}
$$

for $0 \leq N \leq L_{+}^{\prime}$. This represents the marginal probability of the configuration at sites $-L_{-}+1,-L_{-}+2, \ldots N$; the rest of the sites can take arbitrary values. The semicolon indicates the position of the blockage in the system.

Let $e_{R}(\eta)$ be the number of the $\eta_{1}, \eta_{2}, \ldots, \eta_{N}$ that take the value 1 , i.e. $e_{R}(\eta)=\sum_{i=1}^{N} \eta_{i}$. For $r$ small particles to the right of the blockage are rare and can be treated as excitations; $e_{R}(\eta)$ can be thought of as the (right) excitation number in the system.

Our proof proceeds by induction: Suppose we know all probabilities of the form $\operatorname{Pr}\left(\eta_{-L_{-}+1}, \eta_{-L_{-}+2}, \ldots, \eta_{0} ; \eta_{1}, \ldots, \eta_{N}\right)_{L_{-}, L_{+}^{\prime}}$ to a certain accuracy, namely to order $e_{R}\left(\left\{\eta_{1}, \ldots, \eta_{N}\right\}\right)+$ $k$ in $r$. Then (for $N>1$ ) we can (show that we can) compute all probabilities of the form $\operatorname{Pr}\left(\eta_{-L_{-}+1}, \eta_{-L_{-}+2}, \ldots, \eta_{0} ; \eta_{1}, \ldots, \eta_{N-1}\right)_{L_{-}, L_{+}^{\prime}}$ up to order $e_{R}\left(\left\{\eta_{1}, \ldots, \eta_{N-1}\right\}\right)+k+1$ in $r$.

This is sufficient to prove our lemma, and in fact more general results: for any size system (with $\left.L_{+}^{\prime} \geq N\right)$ we can compute $\operatorname{Pr}\left(\eta_{-L_{-}+1}, \eta_{-L_{-}+2}, \ldots, \eta_{0} ; \eta_{1}, \ldots, \eta_{N}\right)_{L_{-}, L_{+}^{\prime}}$ up to order $e_{R}(\eta)$ simply by making use of the fact that excitations are created at rate $r$, providing the initial step in the induction. This estimate (and as a result all following estimates) is independent of $L_{+}^{\prime}$ and thus also valid for all $L_{+} \geq L_{+}^{\prime}$. Since the current is

$$
J(r)=r \operatorname{Pr}(1 ; 0)=r[1-\operatorname{Pr}(0 ; 0)-\operatorname{Pr}(1 ; 1)-\operatorname{Pr}(0 ; 1)]
$$

if we can iterate the induction step $j$ times we know the current to order $2+j$.

So let us return to the induction step. Consider the transitions that occur between the different values of $\eta_{-L_{-}+1}, \ldots \eta_{N-1}$ : we will give estimates on the rates of these transitions in the stationary 


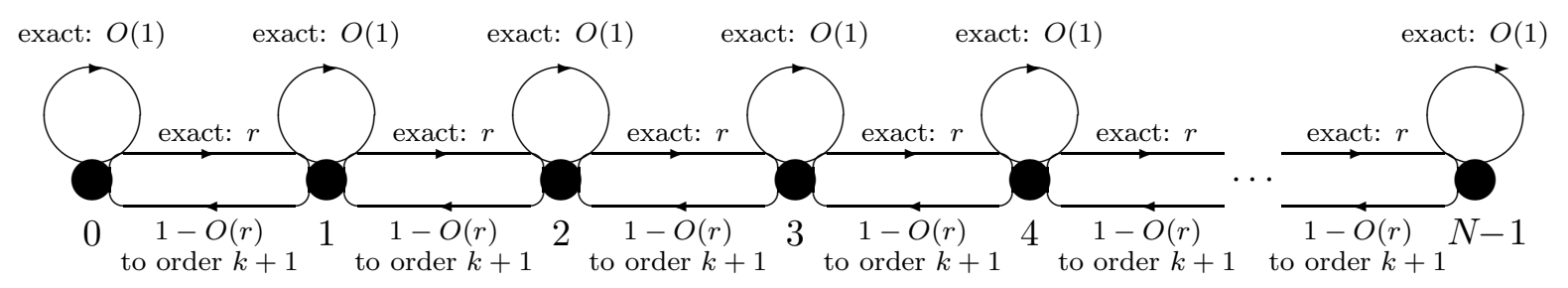

Figure 5: Markov Chain-like representation for the transition rates in the partial system. The indices on the "states" are the $e_{R}(\eta)$ which are altered by the "horizontal" transitions. The "circular" transitions are those between states with the same $e_{R}(\eta)$.

state. (The rate of a transition $\tau \rightarrow \tau^{\prime}$, rate $\left[\tau \rightarrow \tau^{\prime}\right]$, where $\tau$ and $\tau^{\prime}$ are sets of configurations, is

$$
\text { rate }\left[\tau \rightarrow \tau^{\prime}\right]=\lim _{\Delta t \rightarrow 0}(\Delta t)^{-1} \operatorname{Pr}\left(\tau^{\prime} \text { at time } t+\Delta t \mid \tau \text { at time } t\right)
$$

Thus all (elementary) transitions between individual configurations occur at rate 0,1 or $r$.) Transitions that increase $e_{R}\left(\left\{\eta_{1}, \ldots, \eta_{N-1}\right\}\right)$ involve a jump across the blockage, and thus simply have rate $r$. Transitions that keep $e_{R}\left(\left\{\eta_{1}, \ldots, \eta_{N-1}\right\}\right)$ fixed do not involve sites outside of $\left\{-L_{-}+1,-L_{-}+2, \ldots, N-1\right\}$ and so have rates that are simple integers. Transitions that decrease $e_{R}\left(\left\{\eta_{1}, \ldots, \eta_{N-1}\right\}\right)$ require that we know something about site $N$, since the only way to reduce $e_{R}\left(\left\{\eta_{1}, \ldots, \eta_{N-1}\right\}\right)$ is for a particle to move from site $N-1$ to site $N$. So

$$
\begin{aligned}
\text { rate } & {\left[e_{R}\left(\left\{\eta_{1}, \ldots, \eta_{N-2}, 1\right\}\right) \rightarrow e_{R}\left(\left\{\eta_{1}, \ldots, \eta_{N-2}, 0\right\}\right)\right] } \\
& =\frac{\operatorname{Pr}\left(\eta_{-L_{-}+1}, \ldots, \eta_{0} ; \eta_{1}, \ldots, \eta_{N-2}, 1,0\right)_{L_{-}, L_{+}^{\prime}}}{\operatorname{Pr}\left(\eta_{-L_{-}+1}, \ldots, \eta_{0} ; \eta_{1}, \ldots, \eta_{N-2}, 1\right)_{L_{-}, L_{+}^{\prime}}} \\
& =1-\frac{\operatorname{Pr}\left(\eta_{-L_{-}+1}, \ldots, \eta_{0} ; \eta_{1}, \ldots, \eta_{N-2}, 1,1\right)_{L_{-}, L_{+}^{\prime}}}{\operatorname{Pr}\left(\eta_{-L_{-}+1}, \ldots, \eta_{0} ; \eta_{1}, \ldots, \eta_{N-2}, 1,1\right)_{L_{-}, L_{+}^{\prime}}+\operatorname{Pr}\left(\eta_{-L_{-}+1}, \ldots, \eta_{0} ; \eta_{1}, \ldots, \eta_{N-2}, 1,0\right)_{L_{-}, L_{+}^{\prime}}}
\end{aligned}
$$

The numerator of (B.4) is $O\left(e_{R}\left(\left\{\eta_{1}, \ldots, \eta_{N-1}\right\}\right)+1\right)$ and we know it to $O\left(e_{R}\left(\left\{\eta_{1}, \ldots, \eta_{N-1}\right\}\right)+k+1\right)$ by the induction hypothesis. The denominator of $(\overline{\mathrm{B} .4})$ is $O\left(e_{R}\left(\left\{\eta_{1}, \ldots, \eta_{N-1}\right\}\right)\right)$ and we know it to $O\left(e_{R}\left(\left\{\eta_{1}, \ldots, \eta_{N-1}\right\}\right)+k\right)$ by the induction hypothesis. Thus the rate in (B.4) is $1-O(r)$ and we know it to $O\left(e_{R}\left(\left\{\eta_{1}, \ldots, \eta_{N-1}\right\}\right)+k+1\right)$. 
Fig. 5 thus represents our transitions, where we group together states with a common $e_{R}$. It is clear that it is consistent to solve for the probabilities in stationary state of the subsystem represented by fig. 5 to $O\left(e_{R}\left(\left\{\eta_{1}, \ldots, \eta_{N-1}\right\}\right)+k+1\right)$. This completes the induction step and thus the proof of the lemma.

The proof of theorem 2 proceeds in identical fashion. The only difference is that in (B.1) one is limited to considering $0 \leq N<L_{+}^{\prime}$ instead of $0 \leq N \leq L_{+}^{\prime}$, ensuring that the transition rates for changing the excitation number do not depend on the boundary conditions. Thus one can perform the induction step one time less than for the $\alpha=\beta=1$ case and the boundary dependence appears one term earlier in the Taylor series.

\section{Acknowledgments}

We thank F. Alexander, B. Derrida and especially M. Bramson for helpful discussions. We also thank the IHES in Bures-sur-Yvette, where part of this work was done, for its hospitality.

\section{References}

[1] H. Spohn: Large-Scale Dynamics of Interacting Particles Texts and Monographs in Physics, Springer-Verlag, 1991; P. A. Ferrari: "Shocks in the Burgers Equation and the Asymmetric Simple Exclusion Process," preprint RT-MAE-9116 (1991); and references therein.

[2] K. Kawasaki: "Diffusion Constants near the Critical Point for Time-Dependent Ising Models. I,” Phys. Rev. 145, 224-230 (1966).

[3] S. Katz, J. Lebowitz and H. Spohn: "Nonequilibrium Steady States of Stochastic Lattice Gas Models of Fast Ionic Conductors," J. Stat. Phys. 34, 497-537 (1984).

[4] A. de Masi and E. Presutti: Mathematical Methods for Hydrodynamic Limits, Lecture Notes in Mathematics 1501, Springer-Verlag, 1991, and references therein.

[5] S. A. Janowsky and J. L. Lebowitz: "Finite Size Effects and Shock Fluctuations in the Asymmetric Simple Exclusion Process," Phys. Rev. A 45, 618-625 (1992).

[6] W. Dietrich, P. Fulde and I. Peschel, "Theoretical models for superionic conductors," Adv. Phys. 29, 527-605 (1980), and references therein.

[7] K. Nagel and M. Schreckenberg, "A cellular automation model for freeway traffic," J. de physique I 2, 2221 (1992). 
[8] D. E. Wolf and L.-H. Tang: "Inhomogeneous Growth Processes," Phys. Rev. Lett. 65, 1591-1594 (1990).

[9] D. Kandel and D. Mukamel: "Defects, Interface Profile and Phase Transitions in Growth Models," Europhys. Lett. 20, 325-329 (1992).

[10] F. J. Alexander, Z. Cheng, S. A. Janowsky and J. L. Lebowitz: "Shock Fluctuations in the Two-Dimensional Asymmetric Simple Exclusion Process," J. Stat. Phys. 68, 761-785 (1992).

[11] M. Bramson, personal communication, has proven this.

[12] B. Schmittman: "Critical behavior of the driven diffusive lattice gas," Int. J. Mod. Phys. B4, 2269-2306 (1990).

[13] P. Garrido, J. L. Lebowitz, C. Maes and H. Spohn: "Long-range correlations for conservative dynamics," Phys. Rev. A 42, 1954-1968 (1990).

[14] R. Bhagavatula, G. Grinstein, Y. He and C. Jayaprakash: "Algebraic Correlations in Conserving Chaotic Systems," Phys. Rev. Lett. 69, 3483-3486 (1992).

[15] B. Derrida, E. Domany and D. Mukamel: "An exact solution of a one dimensional asymmetric exclusion model with open boundaries," J. Stat. Phys. 69, 667-687 (1992).

[16] J. Krug: "Steady State Selection in Driven Diffusive Systems," in Spontaneous formation of space-time structures and criticality, T. Riste and D. Sherrington, eds., Plenum, 1991.

[17] B. Derrida, M. R. Evans, V. Hakim and V. Pasquier: "Exact Solution of a 1D Asymmetric Exclusion Model Using a Matrix Formulation," J. Phys. A 26, 1493-1517 (1993). 